\title{
Ellipsoidal set-membership state estimation for descriptor systems
}

\author{
Dory Merhy*, Cristina Stoica Maniu*, Teodoro Alamo ${ }^{\dagger}$, Eduardo F. Camacho ${ }^{\dagger}$ \\ * Laboratoire des Signaux et Systèmes (L2S), \\ CentraleSupélec-CNRS-Université Paris-Sud, \\ Université Paris-Saclay, \\ Gif-sur-Yvette, France, F91192 \\ E-mail: dory.merhy@12s.centralesupelec.fr; \\ † Department of Ingeniería de Sistemas y Automática, \\ Universidad de Sevilla, \\ Sevilla, Spain, 41092 \\ E-mail: alamo@cartuja.us.es; \\ eduardo@esi.us.es
}

cristina.stoica@12s.centralesupelec.fr

\begin{abstract}
This paper presents an ellipsoidal set-membership state estimation technique for discrete-time linear time-invariant descriptor systems with bounded perturbations and noises. The approach proceeds off-line by minimizing a parameter with respect to a linear matrix inequality (LMI). The system state of the considered descriptor system is guaranteed to belong to an ellipsoidal set at each time instant. Finally, the proposed technique is analyzed via an illustrative example.
\end{abstract}

Index Terms-descriptor systems, set-membership state estimation

\section{INTRODUCTION}

A mathematical model is in general required for the investigation of systems dynamics via analysis and simulation. Usually, the behavior of a dynamical system is described by differential equations. However, many physical systems are modeled using differential equations coupled with algebraic equations to take into account the conservation laws (mass, volume or energy conservation laws). Overall, the system can be modeled using a combination of differential and algebraic equations, i.e. by differential-algebraic equations (DAE). In control engineering, these systems are known as descriptor systems or singular systems [1]. The formulation of descriptor systems retains the physical meaning of the modeled system, e.g. mechanical systems taking into account constraints related to contact phenomena [2]. It is therefore useful in electrical networks [3], power [4], chemical [5], biological [6] and economic [2] systems, aircraft modeling [7] etc. In the last few decades, the development of reliable studies for the stability [8], controllability [9], [10] and observability [11], [12] of descriptor systems have been the center of attention for more and more researchers. The need of these studies arises from the fact that descriptor systems offer a more general overview of the system description than the standard state-space system. Indeed, when the descriptor matrix is the identity matrix, the descriptor representation is equivalent to the standard statespace representation [13]. In order to make descriptor systems accessible to expert or non-specialist users, a descriptor system toolbox for Matlab has been introduced in [14] providing new tools to manipulate generalized state-space systems, both for the case of continuous- and discrete-time systems. In addition, this toolbox proposes numerous extensions for systems with singular descriptor matrix and provides robust tools for fault detection and isolation of descriptor systems.

In control theory, knowing the system state is necessary to solve different control problems, nevertheless the state variables can not always be determined by direct observation [15]. This issue is mainly tackled by state observers that provide an estimate of the state of a given real system, from input and output measurements, taking into consideration possible perturbations and measurement noises [16]. Among the work done in literature, state observers are designed for linear [13], [17] and non-linear descriptor systems [18], [19], [20], [21], [22]. Due to its simplicity and efficiency, the Kalman filter [15] is a powerful tool widely used in stochastic state estimation for classical and descriptor systems [23], [24], [25] via its different versions. The stochastic approaches, including the classical Kalman filter, assume known distribution of the state perturbation and measurement noises.

Alternatively, a growing part of literature has examined (mainly for standard state-space systems) deterministic state estimation techniques that considers more realistic assumptions of unknown but bounded perturbations and measurement noises [26], [27]. These techniques can be classified into two main categories: interval state observers [28], where the state is approximated by its interval hull, and set-membership state estimation techniques [29], where the state belongs to a deterministic set with a known geometrical structure. In set-membership state estimation approaches, the evolution of the system state at each time instant is described by a set consistent with the model of the system, the bounded perturbations and measurement noises simultaneously. Among these sets are, for example, polytopes [30], zonotopes [31], [32], [33] and ellipsoids [34], [35]. Generally, the ellipsoidal set is privileged in literature for its simplicity [36]. Lately, the Kalman filter and a guaranteed ellipsoidal estimation technique were compared in [37], showing a better accuracy for the considered set-membership technique but with higher computational complexity compared to the classical Kalman filter. Various papers discussed merging both stochastic and deterministic approaches in one technique. For instance, a zonotopic Kalman observer has been presented in [38], [39], [40], introducing a new notion of filter covariation for standard 
state-space systems. The same problem is treated in a different manner in [41], where the estimated state via the Kalman filter is constrained by a given zonotope at each time instant. Indeed, the set-membership state estimation techniques were firstly introduced to standard linear time invariant systems, before inspiring similar approaches for descriptor systems. In this context, a zonotopic set-membership state estimation approach has been proposed in [42], [43] for discrete-time descriptor systems subject to uncertainties and unknown inputs.

Despite the fact that set-membership state estimation techniques offer a good estimation accuracy, while maintaining a realistic aspect of the problem, there is a lack of setmembership state estimation approaches for descriptor systems. Therefore, in the present paper, set-membership state estimation techniques are formulated as feasible optimization problems solved to compute guaranteed bounds for the components of the state vector of descriptor systems subject to bounded perturbations and measurement noises. In this context, the main contribution of this paper is to extend the off-line guaranteed ellipsoidal set-membership state estimation approach from [44] for linear time invariant discrete-time descriptor systems. In this context, a constant observer gain matrix for the considered descriptor system is computed offline via a linear matrix inequality (LMI) optimization problem. The radius of the ellipsoidal set estimating the state at each time instant is computed using a very simple equation to avoid high computational complexity. Indeed, using the offline technique the system state of any descriptor system is guaranteed to belong to an ellipsoid at each time instant.

The remainder of the paper proceeds as follows. Section II gives a brief formulation of the estimation problem. Section III presents the off-line ellipsoidal set-membership state estimation technique for linear discrete-time descriptor systems. To illustrate the results, an example is provided in Section IV. Conclusions and perspectives are drawn in the last section.

Notation. Here, $[a, b]$ defined by $\{x \in \mathbb{R}: a \leq x \leq b\}$ denotes an interval. Thus, the box $\mathbf{B}=[-1,1]$ refers to a unitary interval. Throughout the paper, $\mathbf{B}^{n}=\{x \in$ $\left.\left(\left[a_{1}, b_{1}\right], \ldots,\left[a_{n}, b_{n}\right]\right)^{\top}: a_{i}=-1, b_{i}=1, i=1, \ldots, n\right\} \subset \mathbb{R}^{n}$ is a unitary box composed by $n$ unitary intervals. The notation $I_{n}$ is used for the identity matrix of dimensions $n \times n$, while $A^{\top}$ stands for the transpose of the matrix $A$. A symmetric matrix $M=M^{\top} \in \mathbb{R}^{n \times n}$ is called a positive definite matrix, designated by $M \succ 0$, if $z^{\top} M z>0$, for all vector $z \in \mathbb{R}^{n} \backslash\left\{0_{n}\right\}$. Additionally, the notation $\mathscr{E}(P, \bar{x}, \rho)=\{x \in$ $\left.\mathbb{R}^{n_{x}}:(x-\bar{x})^{\top} P(x-\bar{x}) \leq \rho\right\}$ is used to refer to the ellipsoid with $\bar{x}$ as center, $\rho$ its so called radius and $P=P^{\top} \succ 0$ its shape matrix. The symbol $*$ is used to denote symmetrical terms of a linear matrix inequality.

\section{Problem Statement}

Let us consider the following discrete-time linear time invariant descriptor system described by the following equations

$$
\left\{\begin{aligned}
E x_{k+1} & =A x_{k}+B u_{k}+D w_{k}, \\
y_{k} & =C x_{k}+F w_{k},
\end{aligned}\right.
$$

where $x_{k} \in \mathbb{R}^{n_{x}}$ is the state vector, $u_{k} \in \mathbb{R}^{n_{u}}$ the input vector and $y_{k} \in \mathbb{R}^{n_{y}}$ the output vector at time instant $k$. Here, the perturbations are unknown but bounded by unitary boxes such that $w_{k} \in \mathbf{B}^{n_{w}}$. Moreover, appropriate dimensions are considered for the system matrices $E \in \mathbb{R}^{n_{x} \times n_{x}}, A \in \mathbb{R}^{n_{x} \times n_{x}}$, $B \in \mathbb{R}^{n_{x} \times n_{u}}, C \in \mathbb{R}^{n_{y} \times n_{x}}, D \in \mathbb{R}^{n_{x} \times n_{w}}$ and $F \in \mathbb{R}^{n_{y} \times n_{w}}$. Besides, the initial state belongs to the ellipsoid

$$
\mathscr{E}\left(P_{0}, \bar{x}_{0}, \rho_{0}\right)=\left\{x \in \mathbb{R}^{n_{x}}:\left(x-\bar{x}_{0}\right)^{\top} P_{0}\left(x-\bar{x}_{0}\right) \leq \rho_{0}\right\},
$$

with $P_{0}=P_{0}^{\top} \succ 0$ being the shape matrix, $\bar{x}_{0}$ the center and $\rho_{0}$ the so called radius. In a descriptor system, $E$ is a possible singular matrix with

$$
\operatorname{rank}(E) \leq n_{x}
$$

Notice that if $E=I_{n_{x}}$, then (1) leads to the standard state-space representation. In order to guarantee the observability of the descriptor system (1), it is assumed that

$$
\operatorname{rank}\left[\begin{array}{l}
E \\
C
\end{array}\right]=n_{x}
$$

and

$$
\operatorname{rank}\left[\begin{array}{c}
\lambda E-A \\
C
\end{array}\right]=n_{x}, \forall \lambda \in \mathbb{C} .
$$

Expressions (4) and (5), guaranteeing infinite observability and finite observability, respectively (see [1], [2]), are needed to ensure the existence of Luenberger type observers for a descriptor system ([1], [45]). Additionally, since (4) holds, there exists two matrices $T \in \mathbb{R}^{n_{x} \times n_{x}}$ and $N \in \mathbb{R}^{n_{x} \times n_{y}}$ such that

$$
T E+N C=I_{n_{x}} .
$$

We now show that this equality allows us to compute $x_{k+1}$ from $x_{k}, u_{k}, w_{k}$ and $w_{k+1}$. Multiplying by $T$ the first equation of system (1) and by $N$ the second equation (evaluated at $k+1)$, we obtain

$$
\left\{\begin{aligned}
T E x_{k+1} & =T A x_{k}+T B u_{k}+T D w_{k} \\
N y_{k+1} & =N C x_{k+1}+N F w_{k+1} .
\end{aligned}\right.
$$

Equivalently,

$$
\left\{\begin{array}{l}
T E x_{k+1}=T A x_{k}+T B u_{k}+T D w_{k}, \\
N C x_{k+1}=N y_{k+1}-N F w_{k+1} .
\end{array}\right.
$$

Adding both equalities, and taking into account the equality $T A+N C=I_{n_{x}}$, we obtain

$$
x_{k+1}=T A x_{k}+T B u_{k}+N y_{k+1}+T D w_{k}-N F w_{k+1} .
$$

If the system (1) is used for control purposes, its controlability has to be assumed

$$
\operatorname{rank}\left[\begin{array}{ll}
E & B
\end{array}\right]=n_{x}
$$

and

$$
\operatorname{rank}[\lambda E-A \quad B]=n_{x}, \forall \lambda \in \mathbb{C} .
$$

Conditions (4)-(8) guarantees a weakly minimal descriptor realization. In the general case, a minimal descriptor realization can be considered by ensuring $A \mathscr{N}(E) \subseteq \mathscr{R}(E)$, where $\mathscr{N}(E)$ 
and $\mathscr{R}(E)$ are the kernel and the range of the descriptor matrix $E$, respectively ([2]).

In this framework, this paper investigates set-membership state estimation approaches based on ellipsoids for the descriptor system (1). Given an ellipsoidal estimation $\mathscr{E}\left(P, \bar{x}_{k}, \rho_{k}\right)$ for the state $x_{k}$ from (1) at time instant $k$, the aim is to find an ellipsoidal estimation for the state $x_{k+1}$ at time instant $k+1$ of the form $\mathscr{E}\left(P, \bar{x}_{k+1}, \rho_{k+1}\right)$. This problem is further addressed in Section III where we detail an off-line guaranteed ellipsoidal state estimation technique for descriptor systems.

\section{OfF-Line GuARANTEEd Ellipsoidal State ESTIMATION FOR DESCRIPTOR SYSTEMS}

In a typical set-membership state estimation problem, the state estimation is done by intersecting the prediction state set and the output strip based on the dynamical model (1). Here, ellipsoids are used for implementing the set-membership approach. Hence, the exact intersection between an ellipsoid and a strip is difficult to compute. This reason motivates the use (during the correction phase) of an outer approximation of the intersection between an ellipsoid and a strip by an ellipsoid.

This section details the guaranteed ellipsoidal state estimation for the discrete-time descriptor system (1). Knowing that the perturbations and noises are bounded, the proposed approach minimizes the radius of the ellipsoidal estimation in such way that, at each time instant $k$, the following inequality is satisfied

$$
\rho_{k+1} \leq \beta \rho_{k}+\sigma
$$

where $\beta \in(0,1)$ is a real bounded number and $\sigma$ is a strictly positive scalar $(\sigma>0)$. We notice that (10) is equivalent to

$$
\rho_{k+1}-\rho_{k} \leq(\beta-1) \rho_{k}+\sigma .
$$

Since $\beta-1<0$, we infer that

$$
\rho_{k+1} \leq \rho_{k}
$$

for every

$$
\rho_{k} \geq \frac{\sigma}{1-\beta}
$$

With a pair of matrices $T$ and $N$ satisfying (6), a Luenberger observer of the following form [46] is designed for the descriptor system (1)

$$
\bar{x}_{k+1}=T A \bar{x}_{k}+T B u_{k}+L\left(y_{k}-C \bar{x}_{k}\right)+N y_{k+1},
$$

where $L$ is the observer gain to be determined such that the error between the real state and the nominal estimated state $\bar{x}_{k}$ asymptotically converges to zero. Guaranteeing the ellipsoidal state estimation for the state vector $x_{k}$ at each time instant $k$ is shown in the following theorem, allowing to extend the results from [44] to the considered descriptor system (1).

Theorem 1. Given a scalar $\beta \in(0,1)$, matrices $T$ and $N$ satisfying (6), and an initial state $x_{0}$, suppose that there exist a matrix $P \in \mathbb{R}^{n_{x} \times n_{x}}$, with $P=P^{\top} \succ 0$, a matrix $Y \in \mathbb{R}^{n_{x} \times n_{y}}$, and a scalar $\sigma>0$ such that the linear matrix inequality

$$
\left[\begin{array}{ccc}
\beta P & * & * \\
0 & \sigma & * \\
P T A-Y C & (P T D-Y F) w_{a}-P N F w_{b} & P
\end{array}\right] \succ 0,
$$

holds for all $w_{a}, w_{b} \in \boldsymbol{B}^{n_{w}}$. Then, the system state $x_{k}$ of the descriptor system (1) satisfies

$$
x_{k} \in \mathscr{E}\left(P, \bar{x}_{k}, \rho_{k}\right), \forall k \geq 0,
$$

where, defining $L=P^{-1} Y, \bar{x}_{0}=x_{0}, \rho_{0}=\bar{x}_{0}^{\top} P \bar{x}_{0}$, the sequence $\bar{x}_{k+1}$ and $\rho_{k+1}, k=0,1 \ldots$, can be obtained from the recursive expressions

$$
\begin{aligned}
\bar{x}_{k+1} & =T A \bar{x}_{k}+T B u_{k}+N y_{k+1}+L\left(y_{k}-C \bar{x}_{k}\right), \\
\rho_{k+1} & =\beta \rho_{k}+\sigma .
\end{aligned}
$$

Proof. Since $T$ and $N$ satisfy (6), we have in virtue of equation (7) that the state vector $x_{k+1}$ of the descriptor system (1) satisfies

$$
x_{k+1}=T A x_{k}+T B u_{k}+N y_{k+1}+T D w_{k}-N F w_{k+1},
$$

The estimation error $z_{k}$ is defined as the difference between the real value of the state $x_{k}$ and the nominal estimated state $\bar{x}_{k}$ at time instant $k$, i.e. $z_{k}=x_{k}-\bar{x}_{k}$. Using (14) and (15), the error dynamic equation is given by

$$
\begin{aligned}
z_{k+1} & =T A z_{k}+T D w_{k}-N F w_{k+1}-L\left(y_{k}-C \bar{x}_{k}\right) \\
& =(T A-L C) z_{k}+(T D-L F) w_{k}-N F w_{k+1} \\
& =A_{L} z_{k}+\eta_{k}
\end{aligned}
$$

with

$$
\begin{aligned}
A_{L} & =T A-L C, \\
\eta_{k} & =(T D-L F) w_{k}-N F w_{k+1} .
\end{aligned}
$$

We notice that, for every $k$,

$$
\eta_{k} \in \Upsilon=\left\{(T D-L F) w_{a}-N F w_{b}: w_{a} \in \mathbf{B}^{n_{w}}, w_{b} \in \mathbf{B}^{n_{w}}\right\} .
$$

To validate Theorem 1 , we prove that if $z_{k}^{\top} P z_{k} \leq \rho_{k}$, then

$$
z_{k+1}^{\top} P z_{k+1}=\left(A_{L} z_{k}+\eta_{k}\right)^{\top} P\left(A_{L} z_{k}+\eta_{k}\right) \leq \beta \rho_{k}+\sigma, \forall \eta_{k} \in \Upsilon .
$$

Denoting

$$
\begin{aligned}
F_{0}\left(z_{k}, \eta_{k}\right) & =\beta \rho_{k}+\sigma-\left(A_{L} z_{k}+\eta_{k}\right)^{\top} P\left(A_{L} z_{k}+\eta_{k}\right), \\
F_{1}\left(z_{k}\right) & =\rho_{k}-z_{k}^{\top} P z_{k},
\end{aligned}
$$

and using the S-Procedure [47], we have that (17) holds if there exists $\mu>0$ such that

$$
F_{0}\left(z_{k}, \eta_{k}\right)-\mu F_{1}\left(z_{k}\right) \geq 0, \forall z_{k} \in \mathbb{R}^{n_{x}}, \forall \eta_{k} \in \Upsilon .
$$

Choosing $\mu=\beta$, and using the definitions of $F_{0}\left(z_{k}, \eta_{k}\right)$ and $F_{1}\left(z_{k}\right)$, we obtain that (17) holds if for every $z_{k} \in \mathbb{R}^{n_{x}}$ and every $\eta_{k} \in \Upsilon$ :

$$
\sigma-\left(A_{L} z_{k}+\eta_{k}\right)^{\top} P\left(A_{L} z_{k}+\eta_{k}\right)+\beta z_{k}^{\top} P z_{k} \geq 0 .
$$


This can be rewritten in matrix form as

$$
\left[\begin{array}{c}
z_{k} \\
1
\end{array}\right]^{\top}\left[\begin{array}{cc}
-A_{L}^{\top} P A_{L}+\beta P & -A_{L}^{\top} P \eta_{k} \\
-\eta_{k}^{\top} P A_{L} & \sigma-\eta_{k}^{\top} P \eta_{k}
\end{array}\right]\left[\begin{array}{c}
z_{k} \\
1
\end{array}\right] \geq 0,
$$

for every $z_{k} \in \mathbb{R}^{n}$ and every $\eta_{k} \in \Upsilon$. This is satisfied if

$$
\left[\begin{array}{cc}
-A_{L}^{\top} P A_{L}+\beta P & -A_{L}^{\top} P \eta_{k} \\
-\eta_{k}^{\top} P A_{L} & \sigma-\eta_{k}^{\top} P \eta_{k}
\end{array}\right] \succ 0, \forall \eta_{k} \in \Upsilon .
$$

Equivalently,

$$
\left[\begin{array}{cc}
\beta P & 0 \\
0 & \sigma
\end{array}\right]-\left[\begin{array}{c}
A_{L}^{\top} P \\
\eta_{k}^{\top} P
\end{array}\right] P^{-1}\left[\begin{array}{cc}
P A_{L} & P \eta_{k}
\end{array}\right] \succ 0, \forall \eta_{k} \in \Upsilon .
$$

The Schur complement [47] applied to the previous equation leads to the following equivalent LMI

$$
\left[\begin{array}{ccc}
\beta P & 0 & A_{L}^{\top} P \\
0 & \sigma & \eta_{k}^{\top} P \\
P A_{L} & P \eta_{k} & P
\end{array}\right] \succ 0, \forall \eta_{k} \in \Upsilon
$$

From the equality $A_{L}=T A-L C$, and the definition of $\Upsilon$ we obtain that (17) is satisfied if for every $w_{a} \in \mathbf{B}^{n_{w}}$ and every $w_{b} \in \mathbf{B}^{n_{w}}$

$$
\left[\begin{array}{ccc}
\beta P & * & * \\
0 & \sigma & * \\
P T A-Y C & (P T D-Y F) w_{a}-P N F w_{b} & P
\end{array}\right] \succ 0
$$

with

$$
Y=P L
$$

This proves the claim of the theorem.

The reader will notice that, in practice, LMI (13) is verified for every $w_{a} \in \mathbf{B}^{n_{w}}$ and every $w_{b} \in \mathbf{B}^{n_{w}}$, if it holds for the set of vertices of the considered unitary boxes.

The center of the ellipsoid is calculated using (12) which is a special form of the Luenberger observer for linear discretetime descriptor systems, with the gain $L=P^{-1} Y$ found after solving the linear matrix inequality (13). Considering the worst case where $\rho_{k+1}=\beta \rho_{k}+\sigma$ at each iteration is a sufficient condition to guarantee the convergence of the sequence. Reducing the size of the associated ellipsoid can be done by minimizing $\sigma$ subject to the LMI (13). Solving this LMI off-line to get a constant matrix gain significantly reduces the computation time of the technique. When we consider that the scalar $\beta$ is a real variable, the expression (13) is a case of a bilinear matrix inequality (BMI). However, since $\beta \in(0,1)$ is a bounded variable, the BMI is rewritten as a linear matrix inequality (LMI) problem by fixing the value of the scalar $\beta$ successively using either the bisection algorithm or an available BMI solver: penbmi solver, for example [48].

In the next section, an illustrative example is considered to show the performance of the proposed ellipsoidal state estimation approaches.

\section{ILlustrative EXAMPLE}

Consider the discrete-time linear time-invariant descriptor system (1)

$$
\left\{\begin{aligned}
E x_{k+1} & =A x_{k}+B u_{k}+D w_{k} \\
y_{k} & =C x_{k}+F w_{k}
\end{aligned}\right.
$$

with the following system matrices:

$$
\begin{aligned}
& E=\left[\begin{array}{lll}
1 & 0 & 0 \\
0 & 1 & 0 \\
0 & 0 & 0
\end{array}\right], A=\left[\begin{array}{ccc}
0.5 & 0 & 0 \\
0.8 & 0.95 & 0 \\
-1 & 0.5 & 1
\end{array}\right], B=\left[\begin{array}{ll}
1 & 0 \\
0 & 1 \\
0 & 0
\end{array}\right], \\
& C=\left[\begin{array}{ccc}
1 & 0 & 1 \\
1 & -1 & 0
\end{array}\right], D=\left[\begin{array}{ccccc}
0.1 & 0 & 0 & 0 & 0 \\
0 & 1.5 & 0 & 0 & 0 \\
0 & 0 & 0.6 & 0 & 0
\end{array}\right], \text { and } \\
& F=\left[\begin{array}{lllll}
0 & 0 & 0 & 0.5 & 0 \\
0 & 0 & 0 & 0 & 1.5
\end{array}\right] .
\end{aligned}
$$

The input signal is $u=\left[\begin{array}{ll}0.5 \sin (t)+1 & -2 \cos (t)\end{array}\right]^{\top}$, for $t \in$ $\left[\begin{array}{ll}0 & 5 \pi\end{array}\right]$, with 50 sampling steps. The perturbation vector $w_{k}$ is randomly generated with $\left\|w_{k}\right\|_{\infty} \leq 1$. The matrices $E, A, B$ and $C$ satisfy the rank conditions (4), (5), (8), (9). A possible solution satisfying condition (6) is

$$
T=\left[\begin{array}{ccc}
0.6667 & 0.3333 & 0 \\
0.3333 & 0.6667 & 0 \\
-0.6667 & -0.3333 & 0
\end{array}\right] \text { and } N=\left[\begin{array}{cc}
0 & 0.3333 \\
0 & -0.3333 \\
1 & -0.3333
\end{array}\right] \text {. }
$$

The initial state belongs to the ellipsoid ${ }^{1} \mathscr{E}\left(P_{0}, \bar{x}_{0}, \rho_{0}\right)$, with $P_{0}=10^{-9} \cdot I_{3}, \bar{x}_{0}=\left[\begin{array}{lll}0 & 0 & 0\end{array}\right]^{\top}$, and $\rho_{0}=10^{-9}$ as an arbitrary initialization.

The off-line technique is tested and analyzed on the considered descriptor system. Simulation results are obtained with an Intel Core $i 7-8750 G 3.10 \mathrm{GHz}$, using the LMI solver mincx of Matlab Robust Control Toolbox.

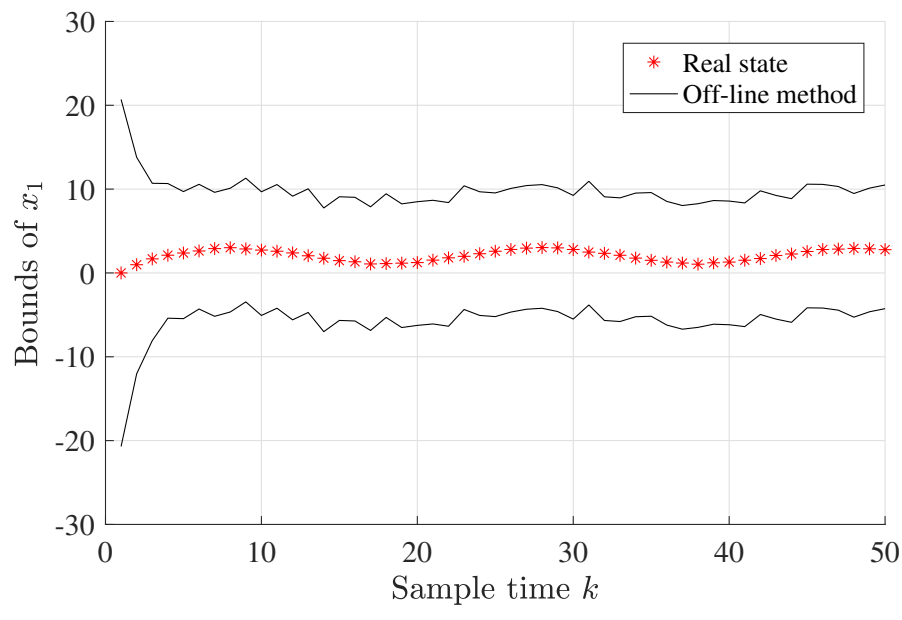

Fig. 1. Bounds on $x_{1}$

Simulation results plotted in Figs. 1-3 illustrate the bounds (black lines) of each element $x_{1}, x_{2}$ and $x_{3}$ of the state vector after 50 iterations of the off-line ellipsoidal set-membership state estimation technique, when solving LMI (13).

Illustrations show that the real state of the descriptor system represented by the red stars is guaranteed to belong to the

\footnotetext{
${ }^{1}$ An arbitrary large ellipsoid is considered for the initialization.
} 


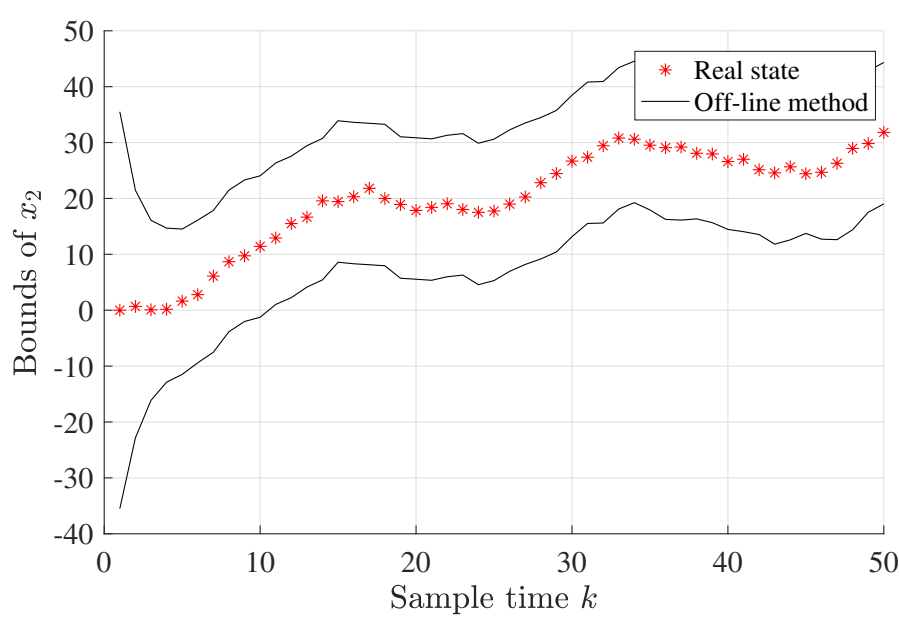

Fig. 2. Bounds on $x_{2}$

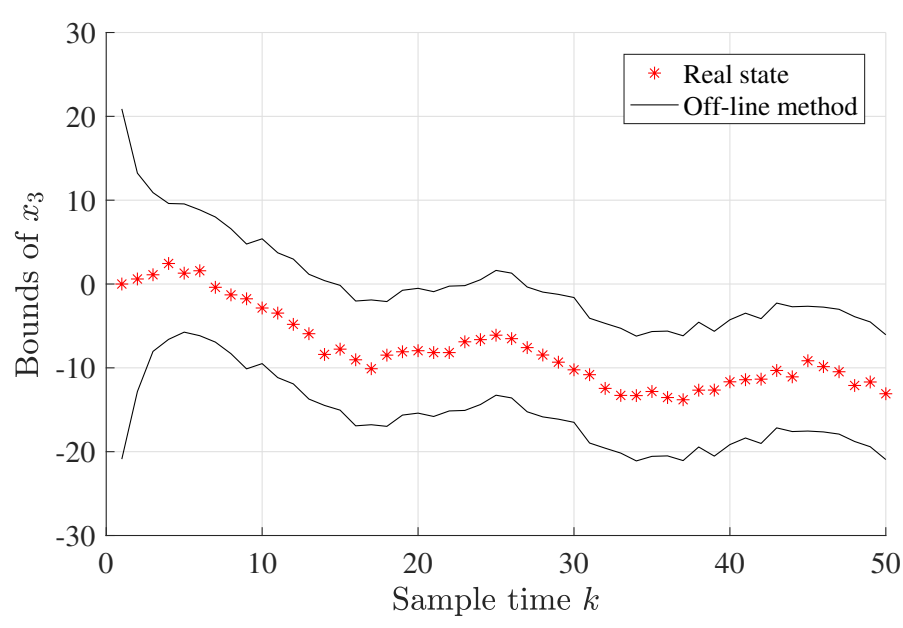

Fig. 3. Bounds on $x_{3}$

bounds computed by the proposed technique at each time instant $k$. The accuracy of this technique can be described by the width of the ellipsoidal bounds shown in the figures, that are in this case relatively large because the goal of this technique is to find a state estimation set with very reduced computation time. Indeed, in this simulation test, the elapsed CPU time for the off-line ellipsoidal technique is 0.61 second (see Table I).

TABLE I

CHARACTERISTICS OF THE OFF-LINE TECHNIQUE

\begin{tabular}{lc} 
Characteristics & Off-line method \\
\hline Simulation time & 0.61 second \\
Volume of the estimation at steady state & 44.69
\end{tabular}

The low computation time of the proposed technique is the fruit of the off-line computation of the feasible set of the state estimate.

Figure 4 illustrates the volume of the ellipsoid estimating the state of the descriptor system at each time instant. The initial volume (due to the initialization of $x_{0}$ in an ample ellipsoid) is relatively large before it decreases to reach a constant value at steady state which is 44.69 as the characteristics Table I shows. This confirms the fact that the volume of the ellipsoidal estimation is minimized at each time instant. Additionally, the proposed estimation technique offers a good accuracy considering the short time it takes to run.

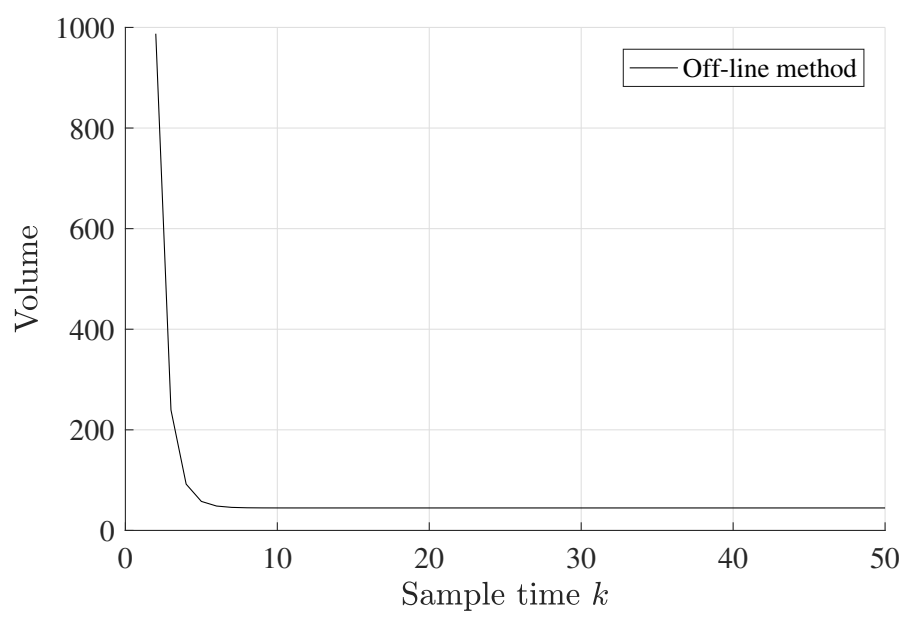

Fig. 4. Volume of the estimation set at steady time

\section{CONCLUSION}

In this paper, we have proposed an extension of an off-line approach of guaranteed ellipsoidal set-membership state estimation technique for discrete-time linear descriptor systems subject to bounded perturbations and noises. The approach minimizes the ellipsoidal set (thus the ellipsoid's volume) estimating the state of the descriptor system once off-line. The technique offers good accuracy and a short computational time.

A promising perspective is to propose a new online ellipsoidal state estimation technique for descriptor systems to improve the accuracy of the proposed off-line method, while keeping the efficiency represented by the computational time. Another perspective is to extend these techniques for descriptor systems with interval uncertainties and apply them in the fault detection context.

\section{ACKNOWLEDGEMENT}

The authors acknowledge MINERCO, FEDER funds, and EU Programme H2020 for funding projects DPI2016-76493C3-1-R and SI-1838/24/2018.

\section{REFERENCES}

[1] L. Dai, Singular control systems. Berlin, Heidelberg: Springer-Verlag, 1989.

[2] A. Varga, Solving Fault Diagnosis Problems - Linear Synthesis Techniques. Springer, 2017.

[3] T. Reis, "Circuit synthesis of passive descriptor systems-a modified nodal approach," International Journal of Circuit Theory and Applications, vol. 38, no. 1, pp. 44-68. 
[4] D. J. Hill and I. M. Mareels, "Stability theory for differential/algebraic systems with application to power systems," IEEE Transactions on Circuits and Systems, vol. 37, no. 11, pp. 1416-1423, 1990.

[5] L. T. Biegler, S. L. Campbell, and V. Mehrmann, Control and optimization with differential-algebraic constraints. SIAM, 2012.

[6] P. Liu, Q. Zhang, X. Yang, and L. Yang, "Passivity and optima control of descriptor biological complex systems," IEEE Transactions on Automatic Control, vol. 53, pp. 122-125, 2008.

[7] B. L. Stevens, F. L. Lewis, and E. N. Johnson, Aircraft control and simulation: dynamics, controls design, and autonomous systems. John Wiley \& Sons, 2015

[8] Q.-L. Han, "A descriptor system approach to robust stability of uncertain neutral systems with discrete and distributed delays," Automatica, vol. 40, no. 10, pp. 1791-1796, 2004

[9] D. Bender and A. Laub, "The linear-quadratic optimal regulator for descriptor systems," IEEE Transactions on Automatic Control, vol. 32 no. 8, pp. 672-688, 1987.

[10] A. Varga, "On stabilization methods of descriptor systems," Systems and Control Letters, vol. 24, no. 2, pp. 133-138, 1995

[11] S. L. Campbell, N. K. Nichols, and W. J. Terrell, "Duality, observability, and controllability for linear time-varying descriptor systems," Circuits, Systems and Signal Processing, vol. 10, no. 4, pp. 455-470, 1991.

[12] E. Yip and R. Sincovec, "Solvability, controllability, and observability of continuous descriptor systems," IEEE Transactions on Automatic Control, vol. 26, no. 3, pp. 702-707, 1981 .

[13] Z. Wang, Y. Shen, X. Zhang, and Q. Wang, "Observer design for discrete-time descriptor systems: an LMI approach," Systems \& Control Letters, vol. 61, no. 6, pp. 683-687, 2012.

[14] A. Varga, "A descriptor systems toolbox for MATLAB," in IEEE International Symposium on Computer-Aided Control System Design, 2000, pp. 150-155.

[15] R. E. Kalman, "A new approach to linear filtering and prediction problems," Transactions of the ASME-Journal of Basic Engineering, vol. 82, no. Series D, pp. 35-45, 1960

[16] D. Luenberger, "Observers for multivariable systems," IEEE Transactions on Automatic Control, vol. 11, no. 2, pp. 190-197, 1966

[17] M. Darouach and M. Boutayeb, "Design of observers for descriptor systems," IEEE transactions on Automatic Control, vol. 40, no. 7, pp. 1323-1327, 1995.

[18] D. Koenig, B. Marx, and D. Jacquet, "Unknown input observers for switched nonlinear discrete time descriptor systems," IEEE Transactions on Automatic Control, vol. 53, no. 1, p. 373, 2008.

[19] D. N. Shields, "Observer design and detection for nonlinear descriptor systems," International Journal of Control, vol. 67, no. 2, pp. 153-168, 1997.

[20] L. Wu, P. Shi, and H. Gao, "State estimation and sliding-mode control of markovian jump singular systems," IEEE Transactions on Automatic Control, vol. 55, no. 5, pp. 1213-1219, 2010.

[21] B. Marx, D. Koenig, and J. Ragot, "Design of observers for TakagiSugeno descriptor systems with unknown inputs and application to fault diagnosis," IET Control Theory Applications, vol. 1, no. 5, pp. 1487 1495, 2007.

[22] V. Estrada-Manzo, Z. Lendek, and T.-M. Guerra, "Unknown input estimation for nonlinear descriptor systems via LMIs and Takagi-Sugeno models," 2015 54th IEEE Conference on Decision and Control, pp. 6349-6354, 2015.

[23] J. Y. Ishihara, M. H. Terra, and J. C. Campos, "Robust Kalman filter for descriptor systems," IEEE Transactions on Automatic Control, vol. 51 no. 8, pp. 1354-1354, 2006.

[24] R. Nikoukhah, S. L. Campbell, and F. Delebecque, "Kalman filtering for general discrete-time linear systems," IEEE Transactions on Automatic Control, vol. 44, no. 10, pp. 1829-1839, 1999.

[25] R. Nikoukhah, A. S. Willsky, and B. C. Levy, "Kalman filtering and Riccati equations for descriptor systems," IEEE Transactions on Automatic Control, vol. 37, no. 9, pp. 1325-1342, 1992.

[26] D. P. Bertsekas and I. B. Rhodes, "Recursive state estimation for a setmembership description of uncertainty," vol. 16(2), pp. 117-128, 1971

[27] E. Fogel and Y. F. Huang, "On the value of information in system identification-bounded noise case," Automatica, vol. 18, 1982.

[28] M. Pourasghar, V. Puig, and C. Ocampo-Martinez, "Comparison of setmembership and interval observer approaches for state estimation of uncertain systems," in Proc. of European Control Conference, Aalborg, Denmark, 2016
[29] F. C. Schweppe, "Recursive state estimation: Unknown but bounded errors and system inputs," IEEE Transaction on Automatic Control, vol. 13(1), pp. 22-28, 1968.

[30] E. Walter and H. Piet-Lahanier, "Exact recursive polyhedral description of the feasible parameter set for bounded-error models," IEEE Transac tion on Automatic Control, vol. 34(8), pp. 911-915, 1989.

[31] T. Alamo, J. M. Bravo, and E. F. Camacho, "Guaranteed state estimation by zonotopes," Automatica, vol. 41, no. 6, pp. 1035-1043, 2005

[32] V. T. H. Le, C. Stoica, T. Alamo, E. F. Camacho, and D. Dumur, "Zonotopic guaranteed state estimation for uncertain systems," Automatica, vol. 49, no. 11, pp. 3418-3424, 2013.

[33] V. T. H. Le, C. Stoica, D. Dumur, T. Alamo, and E. F. Camacho, "Robust tube-based constrained predictive control via zonotopic set-membership estimation," in 50th IEEE Conference on Decision and Control and European Control Conference, 2011, pp. 4580-4585.

[34] A. B. Kurzhanski and I. Vályi, Ellipsoidal calculus for estimation and control. Birkhaüser Boston, 1996.

[35] S. Ben Chabane, C. Stoica Maniu, T. Alamo, E. F. Camacho, and D. Dumur, "Improved set-membership estimation approach based on zonotopes and ellipsoids," in European Control Conference, 2014, pp. 993-998.

[36] V. Puig, "Fault diagnosis and fault tolerant control using set-membership approaches: Application to real case studies," International Journal of Applied Mathematics and Computer Science, vol. 20, no. 4, pp. 619635, 2010.

[37] D. Merhy, C. Stoica Maniu, T. Alamo, E. Camacho, and S. Ben Chabane, "Comparison between two state estimation techniques for linear systems," in 20th IFAC World Congress, no. 4855-4859, Toulouse, France, 2017.

[38] C. Combastel, "Merging Kalman filtering and zonotopic state bounding for robust fault detection under noisy environment," IFACPapersOnLine, vol. 48, no. 21, pp. 289-295, 2015.

[39] M. Pourasghar, C. Combastel, V. Puig, and C. Ocampo-Martinez, "FDZKF: A zonotopic Kalman filter optimizing fault detection rather than state estimation," Journal of Process Control, vol. 73, pp. 89 - 102 , 2019.

[40] C. Combastel, "Zonotopes and Kalman observers: Gain optimality under distinct uncertainty paradigms and robust convergence," Automatica, vol. 55 , pp. $265-273,2015$.

[41] D. Merhy, T. Alamo, C. Stoica Maniu, and E. Camacho, "Zonotopic constrained Kalman filter based on a dual formulation," in IEEE Conference on Decision and Control, 2018, pp. 6396-6401.

[42] Y. Wang, V. Puig, and G. Cembrano, "Set-membership approach and Kalman observer based on zonotopes for discrete-time descriptor systems," Automatica, vol. 93, pp. 435-443, 2018.

[43] Y. Wang, V. Puig, G. Cembrano, and T. Alamo, "Guaranteed state estimation and fault detection based on zonotopes for differentialalgebraic-equation systems," in 3rd Conference on Control and FaultTolerant Systems, 2016, pp. 478-484.

[44] S. Ben Chabane, C. Stoica Maniu, T. Alamo, E. Camacho, and D. Dumur, "A new approach for guaranteed ellipsoidal state estimation," 19th IFAC World Congress, vol. 47, no. 3, pp. 6533 - 6538, 2014.

[45] M. Hou and P. C. Muller, "Design of a class of Luenberger observers for descriptor systems," IEEE Transactions on Automatic Control, vol. 40, no. 1, pp. 133-136, 1995.

[46] Z. Wang, Y. Shen, X. Zhang, and Q. Wang, "Observer design for discrete-time descriptor systems: An LMI approach," Systems and Control Letters, vol. 61, no. 6, pp. 683 - 687, 2012.

[47] S. Boyd, L. El Ghaoui, E. Feron, and V. Balakrishnan, Linear Matrix Inequalities in System and Control Theory. SIAM, 1994.

[48] M. Kočvara and S. Stingl, "PENNON A Code for convex nonlinear and semidefinite programming," Optimization methods and software, vol. 18(3), pp. 317-333, 2003 